\title{
Gut Microbiota Dysbiosis and Their Impact on Biochemical and Inflammatory Profiles in Cafeteria Diet Fed Sprague Dawley Rats
}

\author{
Reem Moath Alasmar ${ }^{1,2}$, Kavitha Varadharajan ${ }^{*}{ }^{*}$, Muralitharan Shanmugakonar ${ }^{1}$, \\ Hamda A. Al-Naemi ${ }^{1,2}$ \\ ${ }^{1}$ Laboratory Animal Research Centre, Qatar University, Doha, Qatar \\ ${ }^{2}$ Department of Biological and Environmental Sciences, Qatar University, Doha, Qatar \\ Email: *kavithasajani@qu.edu.qa
}

How to cite this paper: Alasmar, R.M., Varadharajan, K., Shanmugakonar, M. and Al-Naemi, H.A. (2019) Gut Microbiota Dysbiosis and Their Impact on Biochemical and Inflammatory Profiles in Cafeteria Diet Fed Sprague Dawley Rats. Advances in Microbiology, 9, 775-789.

https://doi.org/10.4236/aim.2019.99047

Received: August 1, 2019

Accepted: September 1, 2019

Published: September 4, 2019

Copyright $\odot 2019$ by author(s) and Scientific Research Publishing Inc. This work is licensed under the Creative Commons Attribution International License (CC BY 4.0).

http://creativecommons.org/licenses/by/4.0/

\begin{abstract}
Gut microbiota plays a major role in regulating the host metabolism and immune system. However, the structure of microbiome population is altered constantly by diverse factors including diet and environment. In particular, the gut microbiome dynamics is influenced by diet composition and their associated metabolites. Many studies in the recent past reported on diet induced dysbiosis in the gut microbiome, the modulating ratio between Firmicutes and Bacteroidetes plays a central role in maintaining the microbiome diversity in the gut and their abundance regulates obese conditions. Although there are several reports on gut microbial dysbiosis (Firmicutes/ Bacteroidetes) at phylum level but only few of them highlight at the genera level. In the present study, we focused more on the impact of cafeteria diet (CAF) with respect to the gut microbiome richness at the genera level in SD rats. Three weeks old Sprague Dawley (SD) rats were fed with normal chow diet and cafeteria diet (CAF). After 10 weeks, serum, tissue samples (small intestine and cecum), cecum fecal and fecal pellet were collected. Biochemical analysis from serum, Gene expression analysis of pro-inflammatory markers from tissues and microbiome analysis from fecal samples were analysed. CAF diet fed rat in the present study developed obesity with increased body weight, few of them developed the resistance to weight gain (WGR) and these animal shows significantly increased abundance of Bacteriodetes-Prevotella compared to obese animals. This study suggests that detailed research needed to address the contribution of microbiota abundance at the genera level. We further explored the influence of diet induced microbiota changes on immune response; increase in Bacteroidetes leads to increased LPS and this in turn elicit immune reaction in the gut. Moreover, variation in gut microbiota also af-
\end{abstract}


fects the gut barrier function (Tight junction proteins) and increase metabolic endotoxemia that leads to activation of innate immune system and low-grade inflammation. Further studies on these lines targeting the microbiota changes in the gut at the genera level is warranted to gain more knowledge.

\section{Keywords}

Gut Microbiota, Cafeteria Diet, Obesity, Inflammatory Markers, RT-PCR

\section{Introduction}

In recent decade, obesity is increasing worldwide in high proportion, both young and adult population is affected and especially the prevalence is more in the developed countries. Obesity is one of the major global health issues linked to multiple diseases such as hypertension, type 2 diabetes mellitus, non-alcoholic fatty liver disease and cardiovascular disorders [1]. Moreover, the impact of this metabolic disorder is so severe that it affects more than 107.7 million children and more than 603.7 million adults around the world [2]. Obesity is a disorder affecting the metabolic function of the body through defecting various cellular and molecular functions. The main contributor for this syndrome is the energy imbalance; when the energy intake exceeds the expended energy, the system falls into the obese physiology that leads to deposition of unspent energy in the form of fat, which leads to obese phenotype. Qatar, a small nation with excess of oil wealth in the gulf region experiencing the increasing prevalence of obesity, $30 \%$ of younger population of 6 to 11 years old children are obese and overweight. Understanding the factor fueling this high prevalence of obesity in this population is crucial to define a strategy to introduce measures and to decide on a therapeutic intervention to monitor and control the spread of this syndrome. Among the various factors, diet plays a major role in terms of energy intake, easy availability of high sugar and high fat diet contributed for this alarming prevalence in this population. Though many studies reported on the role of diet in obesity, very few reports focus on the role of microbiome on diet and energy homeostasis. Human body enriched with millions of bacteria and especially the diversity in the gut microbiota played a major role in various physiological and immunological mechanisms that leads to signaling events for normal cell function. Maintaining gut microbiota homeostasis is important as the dysbiosis of microbiota in the gut leads to dysfunctional barrier function that is the cause of leaky gut and dysfunctional physiological and signaling pathways. Diet as a major player influencing the gut microbiome population, different types of diet such as high carbohydrate, high fat, dietary fibers, western diet, pre/probiotics and vegetarian diet are mainly studied and found that they have a significant role in shifts/ shaping the gut microbiome within 24 hours of intake.

Microbiome is an integral part of the human body with highest density of bacteria in the gut, ranging from $1 \times 10^{4}$ cells/g in the jejunum to $\left(1 \times 10^{14}\right)$ 
cells/g in the colon. The microbial diversity within the gastro intestinal (GI) tract has been linked to number of metabolic diseases, including diabetes, non-alcoholic fatty liver disease, cardiovascular disease, and obesity. Recent studies observed that $80 \%-90 \%$ of the bacterial phylum types in the human GI tract falls under two main phyla, Bacteroidetes (gram-negative) and Firmicutes (gram-positive), and secondary phyla, Actinobacteria (gram-negative), and Proteobacteria (gramnegative). Various factors affect the microbiota composition and distribution, such as newborn delivery method, antibiotic usage, age, exercise, and disease state; moreover, diet composition seems to be another major factor influencing the microbiota diversity. More consumption of sugar is closely associated with obesity and metabolic diseases [2], however the impact of high sugar diet on the landscape of gut microbiota is still not clear

The dynamic change in the gut microbial population due to the impact of diet is one of the main problems lead to different health disorders. For example, obesity and overweight gain that has a strong relation with inflammation and insulin resistance. In addition, gut microbiota makes significant contribution to host metabolism, by modulating host nutrition and energy harvest as well as production of some vitamins, such as $\mathrm{K}^{+}$, folic acid and B12. The hallmark of obesity is the fat deposition in adipose tissues that induce immune response and that leads to inflammation and changes in tight junction barrier on GI cells [1]. There are reports that, most of the inflammatory processes are a consequence of obesity [3]. In particular, fat and carbohydrate diet plays a major role in the development of insulin resistance and other obesity related features. [4]. Due to obesity and related metabolic stress, the immune receptors activated that triggers the cascade of inflammation reactions.

Understanding the impacts of high sugar and carbohydrate diet on gut micobiota and their contribution for obese phenotype is critical to appreciate the influence of gut microbiota on metabolic dysfunction. There are reports concludes that, a pro-inflammatory microbiota community has been observed, their contribution is significant in inducing various metabolic syndrome and supporting a causal relationship between bacterial induced inflammation and metabolic disorders [4]. Impact of gut microbiota on the GI tract permeability leads to leaky gut syndrome, high carbohydrate diet induce many changes in the gut microbiota diversity that leads to changes in the intestinal permeability and dysregulate the leakage of LPS into circulation by decreasing the expression of intestinal tight junction proteins.

A leaky gut allows easy translocation of pro-inflammatory mediators into blood circulation and the bacterial LPS reaches the liver through the portal circulation instigating inflammation and abnormal lipid accumulation in several tissues. The inflammations due to bacterial derived LPS mainly have high levels of inflammatory cytokines (tumor necrosis factor alpha (TNF- $\alpha$ ), monocyte chemoattractant protein 1 (MCP1), interleukin 1 beta (IL-1 $\beta$ ), interleukin 6 (IL-6), interleukin 10 (IL-10), interleukin 22 (IL-22), and lipid synthesis enzymes 
such as acetyl-CoA carboxylase 1, fatty acid synthase (FAS), and sterol regulatory element-binding protein 1 (SREBP1)) [5]. Hence, inflammation driven by microbiota is the crucial factor triggering the obesity and other associated pathological conditions. Actually, the abnormal immune signaling plays a main role in microbiota induced metabolic syndrome for example deficiency of TLR5 signaling in rat is sufficient to induce obesity. Although many reports correlates the influence of various diet regime that impacts the microbiota composition [2] in the gut and their eventual dysbiosis leads to metabolic diseases, only few reports highlights the dynamics of their metabolism and metabolite analysis [6].

Along these lines, we attempted to study and analyze the taxonomical abundance present in fecal and cecum samples from the cafeteria diet (CAF) fed young Sprague Dawley (SD) rats compared with normal chow diet fed rats. In the present study, microbial diversity at the genera level explored in experimental animals using molecular methods. In addition, variation in their behavior, phenotype and difference in the metabolic activity between CAF and normal chow diet fed animals also documented in detail.

\section{Materials and Methods}

\subsection{Experimental Design}

Three weeks old, (50 \pm 1.09 g) Sprague Dawley (SD) rats were obtained from breeding male and female SD rat breeders purchased from Charles River, UK and maintained in Laboratory Animal Research Center (LARC), Qatar University (QU), Qatar. Qatar university institutional ethical clearance, animal care and use committee (QU -IACUC) and Institutional Biosafety Committee (IBC) approvals obtained for the study. The rats housed under standard animal husbandry conditions with 24 hours dark and light cycle. Control $(n=18)$ and experimental animal $(\mathrm{n}=18)$ groups were maintained in QU-LARC IVC controlled environment and fed with normal chow diet (Carbohydrate 56.9\%; protein $18.0 \%$ and lipids $4.8 \%$ ) and cafeteria diet (Carbohydrate 66.05\%; Protein 11.04\% and lipids 7.22\%) respectively. Body weight, food and water intake were recorded weekly and phenotype of two groups were monitored thoroughly. The detailed husbandry conditions, experimental design, diet and animal phenotype observations were described in our previous study [6].

\subsection{Sample Collection and Storage}

After completion of study (10 weeks), all experimental animals subjected to fasting for 12 hours, anesthesia induced by an intraperitoneal dose of Thiopentone sodium $(0.04 \mathrm{mg} / \mathrm{kg}$ body weight) and sacrificed for sample collection. Fresh fecal pellets collected from all experimental groups individually for microbiome analysis. After euthanasia, blood samples collected from all experimental animals individually by cardiac puncture and the serum samples were separated by centrifugation for biochemical analysis. After necropsy, the stool samples from cecum, tissue samples of small intestine and cecum were collected from all 
animal groups for microbiome analysis and gene expression analysis of proinflammatory markers. Samples stored at $-80^{\circ} \mathrm{C}$ for further analysis.

\subsection{Biochemical Analysis}

Serum samples were assayed for levels of total cholesterol, low-density lipoprotein (LDL) cholesterol, direct-high-density lipoprotein cholesterol (DHDL), Triglyceride (TGS), Urea, Aspartate transaminase (AST), Alanine transaminase (ALT), Total protein (TP2), Albumin (ALB), Creatinine (CREA) and Glucose Hexokinase (GLUOHK) by using Biochemistry Analyzer (Randox RX Daytona, UK).

\subsection{DNA Extraction from Fecal Sample}

Genomic DNA extracted from cecum stool samples and fecal pellet using QIAamp DNA Stool Mini kit (Qiagen). Before process the samples, the frozen samples were kept on ice for 30 minutes for thawing. The fecal and cecum stool samples were weighed (approximately 180 - $220 \mathrm{mg}$ ) from each experimental groups (Normal and obese groups). Fecal samples were homogenized using buffers provided with DNA extraction kit and the supernatant was collected after centrifugation. Using lysis buffer and proteinase $\mathrm{K}$, the supernatant was subjected to microbial cell lysis followed by the extraction protocol according to the kit instruction. Genomic DNA was extracted using spin column method and lastly eluted by elution buffer. The genomic DNA was quantified by nanophotometer (IMPLEN).

\subsection{Gut Microbiome Analysis Using Real-Time PCR}

Diversity of gut microbiome present in the fecal pellet and the cecum fecal sample was analyzed using SYBR green master mix using specific primers in Quantstudio 6 Flex Real Time PCR system (Applied Biosystem). Primer sequences used for the target 16s rRNA gene of the gut microbiota (Firmicutes, Bacteroidetes and Actinobacteria) described in Table 1 [7]. To verify the specificity of amplicon, a melting curve analysis in addition to agarose gel electrophoresis was

Table 1. Describes the primer sequence details of gut microbiota.

\begin{tabular}{cccc}
\hline s. No. & Target group & Forward Primer sequence $\left(\mathbf{5}^{\prime}-\mathbf{3}^{\prime}\right)$ & Reverse Primer sequence (5'-3') \\
\hline 1 & Firmicutes & ATG TGG TTT AAT TCG AAG CA & AGC TGA CGA CAA CCA TGC AC \\
2 & Bacteroidetes & CAT GTG GTT TAA TTC GAT GAT & AGC TGA CGA CAA CCA TGC AG \\
3 & Lactobacillus & GAG GCA GCA GTA GGG AAT CTT CG & GC CAG TTA CTA CCT CTA TCC TTC TTC \\
4 & Enterococcus & CCC TTA TTG TTA GTT GCC ATC ATT & ACT CGT TGT ACT TCC CT TGT \\
5 & Clostridium & GCA CAA GCA GTG GAG T & CTT CCT CCG TTT TGT CAA \\
6 & Bacteroides & ATA GCC TTT CGA AAG RAA GAT & CCA GTA TCA ACT GCA ATT TTA \\
7 & Prevotella & CAC RGT AAA CGA TGG ATG CC & GGT CGG GTT GCA GAC C \\
8 & Bifidobacterium & CGC GTC YGG TGT GAA AG & CCC CAC ATC CAG CAT CCA
\end{tabular}


performed. The relative intensity of amplicon captured in gel documentation system and calculated by image J analysis software to attain the abundance ratio.

\subsection{RNA Extraction from Intestinal Tissues}

Total RNA was extracted from frozen small intestine and cecum tissue samples using Trizol LS reagent (Invitrogen). Frozen tissue samples were preprocessed individually using liquid nitrogen and followed by Trizol LS reagent according to the manufacturer's instruction. Briefly, homogenized tissues were incubated in Trizol reagent until complete dissociation of nucleoproteins. Aqueous phase separated after addition of chloroform and the total RNA was precipitated with isopropanol and washed in $75 \%$ ethanol to attain RNA pellet. Extracted RNA samples were quantified by nanophotometer (IMPLEN) and stored at $-80^{\circ} \mathrm{C}$ for further analysis.

\section{7. cDNA Synthesis and Gene Expression Analysis}

A known quantity of RNA was used for cDNA synthesis by High Capacity cDNA reverse transcription kit (Applied Biosystem) using Proflex PCR machine (Applied Biosystem). Gene expression analysis carried out using specific Taqman primers for each inflammatory marker (IL-1 $\beta$, IL-10, IL-6, IL-22, $\alpha$-TNF and NFkB) and analyzed using Quantstudio 6 Flex Real-Time PCR system (Applied Biosystem). Gene expression of the respective target genes were compared and normalized against endogenous control (GAPDH) and gene expression in the experimental control samples also determined using Real-Time PCR software (with relative quantification $(\mathrm{RQ})$ expressed on a $\log 10$ scale $(\log R Q)$ to show fold-changes in gene expression).

\subsection{Statistical Analysis}

One-way ANOVA was used to determine the effect of cafeteria diet on the serum parameters and to compare mean between the groups (control and obese). In addition, Tukey's multiple comparison test also done to determine differences between all experimental groups, whenever no significant difference was identified. Dunnett Multiple Comparisons test used to compare control groups with experimental groups. To analyze difference between two groups, a $t$ test was accomplished and the differences were considered statistically significant when $\mathrm{P}$ value $\mathrm{P}<0.05$. All statistical analyses performed using Minitab software.

\section{Results}

During the study period, cafeteria diet fed SD rats gained more weight (Obese group) when compared with normal chow diet fed SD rats (Control group). Interestingly, some of the animals in the cafeteria diet fed groups were not gained their weight and the body weight remain same as control normal chow diet fed rats and they are assigned as weight gain resistant group (WGR). All the experimental animals subjected to overnight fasting and euthanized as per LARC 
standard procedure. After sample collection, the samples were processed and stored at appropriate condition immediately. To asses and correlate the relationship between the diets, gut microbiota and the host immune response several analyses such as biochemical parameters and gene expression of pro-inflammatory markers were carried out.

With respect to the serum biochemical analysis, CAF diet fed rats (Obese and WGR groups) showed significant increase in serum glucose and triglycerides level when compared with the control animals. However, there is no significant difference in the cholesterol, HDL and creatinine profile in all these groups. Obese group showed a minor deviation in urea level whereas the creatinine is in a decreased trend in all the three groups. As far as LDL concerned, obese rats showed less concentration paralleled with other groups.

With regard to the hepatic biomarkers, total protein concentration of obese group as well as in control groups (C) are significantly elevated compared to WGR groups. Level of AST in CAF diet fed rats are higher than the control groups whereas comparing the CAF diet fed rats with WGR groups a significantly higher AST level is found in the obese group. ALT of obese rats showed increased level when compare with control rats whereas WGR rats showed lesser than the control rats. Obese groups showed minor reduction in serum albumin when compared with the other two groups (Control and WGR groups) (Figure $1)$.

Change in the composition of gut microbiota population due to cafeteria diet was analyzed by Real Time PCR method using specific primers. Diet mainly alters the phylum Firmicutes, Bacteroidetes and Actinobacteria in the gut microbial diversity. Among the phylum Firmicutes and Bacteroidetes, predominantly involved genera such as Lactobacillus, Enterococcus and Clostridium (Firmicutes), Bacteroides and Prevotella (Bacteroidetes) and Bifidobacterium (Actinobacteria) were analyzed for the gut microbial dysbiosis. Figure 2(a) and Figure 2(b)

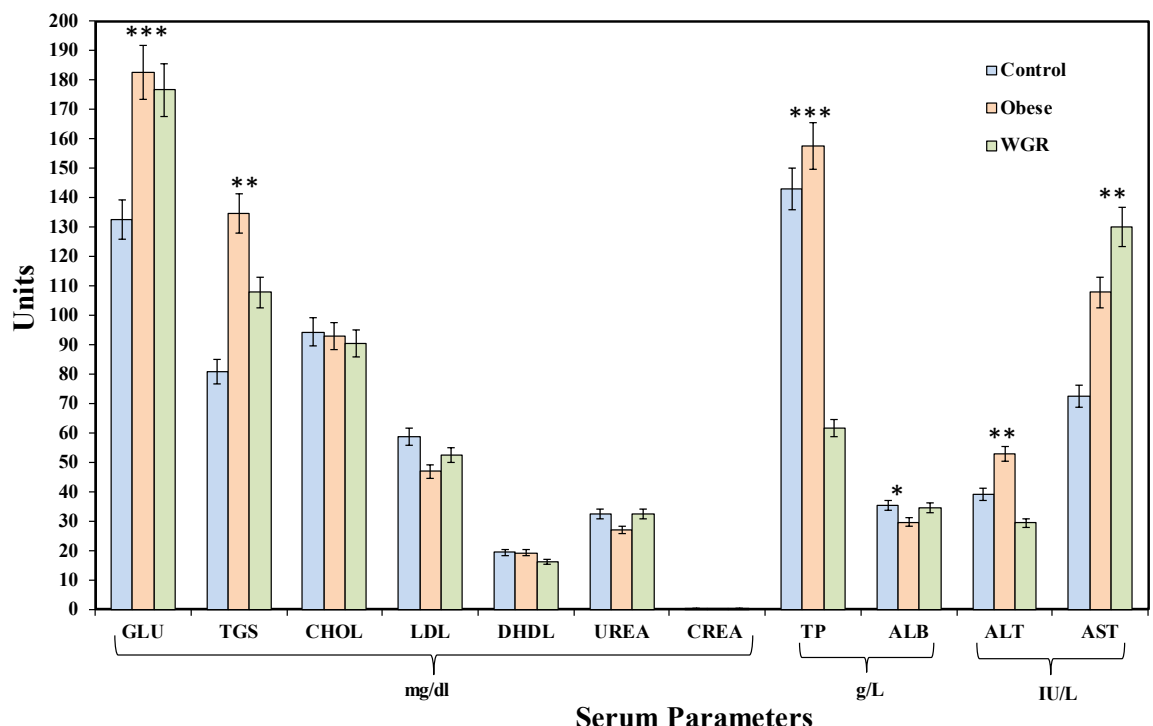

Figure 1. Biochemical parameters of CAF diet and Normal chow diet fed SD rats. 


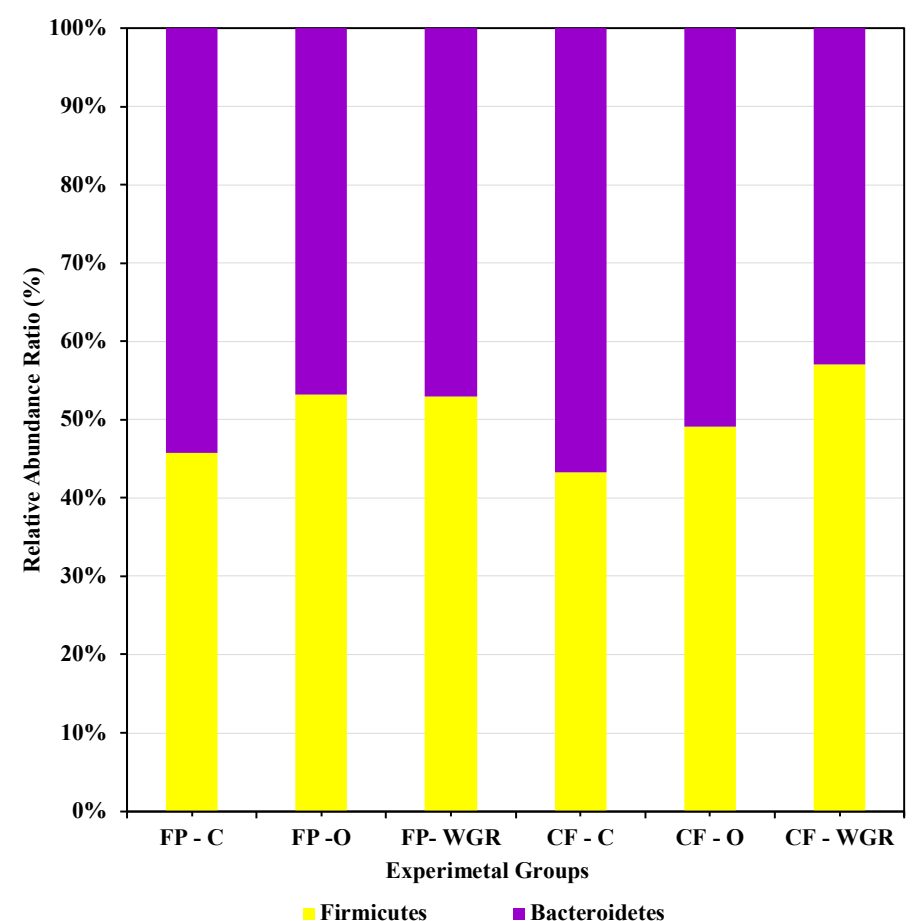

(a)

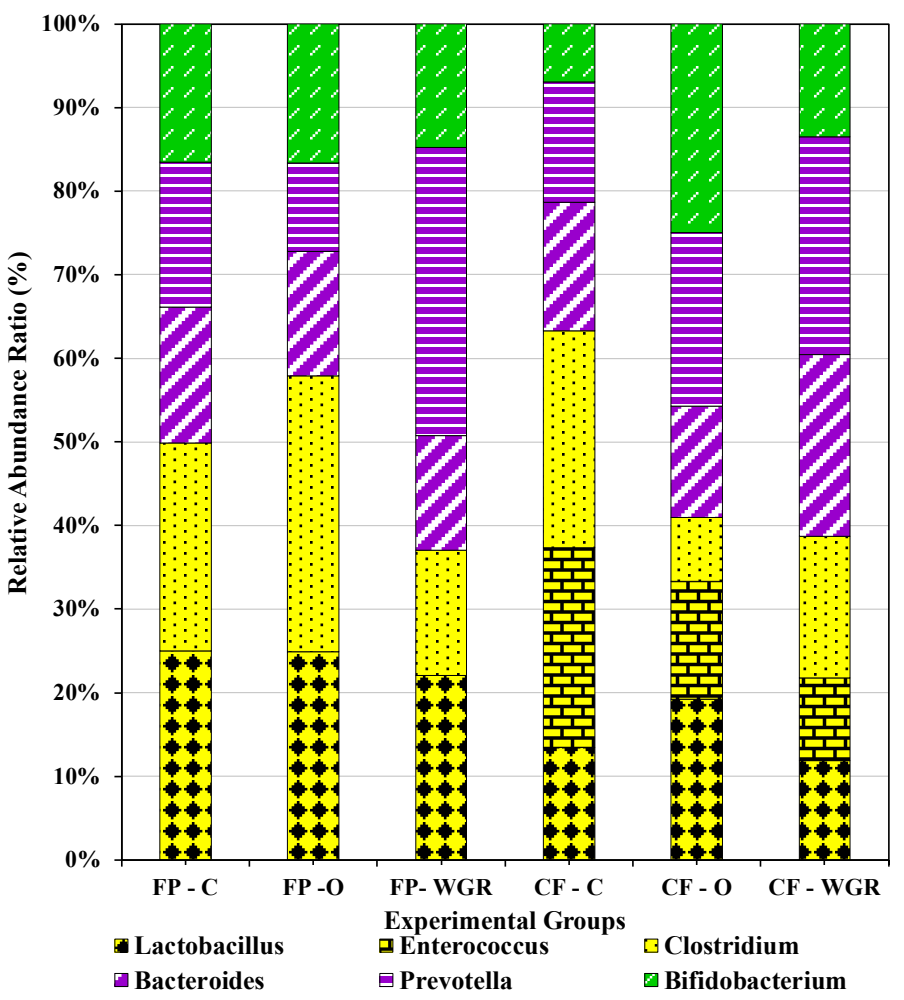

(b)

Figure 2. (a) Phylum level (Firumicutes/ Bacteroidetes) relative abundance ratio of gut microbial population in CAF diet and Normal chow diet fed SD rats; (b) Genera level (Firumicutes/Bacteroidetes) relative abundance ratio of gut microbial population in CAF diet and Normal chow diet fed SD rats. (Firmicutes-Yellow color; Bacteroidetes-Purple color; Actinobacteria-Green color). 
explains the gut microbial dysbiosis in fecal and cecum stool samples from normal chow diet and cafeteria diet fed SD rats. Relative abundance of phylum Bacteriodetes are significantly higher in control group of both fecal and cecum samples. However, Firmicutes is higher than the Bacteroidetes in obese group and weight gain resistant groups. There is a minor alteration in the ratio of Firmicutes/ Bacteroidetes in CAF diet fed animals when compared with their respective controls (Figure 2(a)).

Figure 2(b) describes the relative abundance ratio of genera Lactobacillus, Enterococcus and Clostridium (Firmicutes), Bacteroides and Prevotella (Bacteroidetes) and Bifidobacterium (Actinobacteria). To explore whether the ratio between Firmicutes and the Bacteroidetes in phylum level could be a confounding factor to impact changes at genera level, we made further analysis of microbiota population. In fecal pellet, relative abundance ratio of Clostridium (Firmicutes) is higher in Obese samples (FP-O) however they are markedly lower in weight gain resistant samples (FP-WGR) when compared with control samples (FP-C). There is no significant variation in the ratio of Lactobacillus among fecal pellet samples. There is no evidence for Enterococccus (Firmicutes) in fecal samples. Meanwhile, relative abundance ratio of genera Pervotella (Bacteroidetes) is significantly higher in weight gain resistant sample (FP-WGR) and not much variation in Bacteroides (Bacteroidetes) and Bifidobacteium (Actinobacteria) irrespective of diet in all the groups. In cecum fecal samples, Lactobacillus (Firmicutes) has exhibited very low variations between the groups. Clostridium and Enterococuss (Firmicutes) abundance is higher in the cecum of control samples than the CAF fed rat samples. Proportion of Genera Pervotella (Bacteroidetes) is more in weight gain resistant sample (CP-WGR) than control (CF-C) and obese (CF-O) samples whereas Bacteroides is higher in weight gain resistant groups (CF-WGR) when compared with other groups. Abundance of Bifidobacterium is significantly increased in both obese (CF-O) and weight gain resistant groups (CF-WGR). Overall, Firmicutes relative abundance ratio is significantly dropped in cecum obese (CF-O) and weight gain resistant samples (FP-WGR \& CP-WGR).

To assess the effect of gut microbiota changes on gut barrier function and inflammation, a panel of inflammatory markers were analyzed for their gene expression pattern. Gene expression analysis of inflammatory markers (IL- $1 \beta$, IL-6, $\alpha$-TNF, NFKB IL-10, IL22,) in cecum and small intestine samples analyzed by Taqman Real Time PCR method. The results revealed that most of inflammatory markers in cecum samples were upregulated in both obese and WGR groups whereas all of them were down regulated in small intestine when compared with control samples. In cecum tissue samples, IL1b expressed more in obese sample when compare to the WGR groups. Other proinflamamtory markers such as IL6, $\alpha$-TNF and Nfkb there is no significant gene expression variation irrespective of diet in all the groups. However, mRNA expression of IL10 and IL22 were upregulated in obese groups compared to WGR group. In small intestine samples, the mRNA expression of the inflammatory markers were downre- 
gulated. However, except IL1b and $\alpha$-TNF all other inflammatory markers were expressed more in obese groups when compared with WGR groups (Figure 3 ).

\section{Discussion}

The aim of the present study is to compare the effect of cafeteria diet and normal chow diet on gut microbiota population in the cecum fecal and fecal pellet samples from experimental and control SD rats. The cafeteria diet selected in current study to prove as an informative tool to induce voluntary hyperphagia and enhance overweight/obesity in rats [8]. Several attempts have been made to reveal the diet induced obesity and its associated physiological disorder [9] [10] [11] [12]. Owing to the influence of various factors, exact mechanism behind the diet-induced obesity is still unclear. In this study, we discuss the interrelation between the diet, gut microbiota and altered physiological parameters. The experimental animals with the approximate body weight of ( $50 \pm 1.09 \mathrm{~g})$ were chosen and separated into two experimental groups (Control-Chow diet fed rats and Obese-Cafeteria (CAF) diet fed rats). During the experimental period, the effect of diet on the weight gain, food consumption, physiological and biochemical activities were observed and recorded periodically. Result and discussion of the said parameters were reported detail in our previous study [6]. Before euthanasia, all experimental group (Control, Obese and Weight gain resistant) subjected to overnight fasting as per LARC recommended protocol. All the respective samples collected and processed accordingly for experimental analysis.

The present study revealed that cafeteria diet fed rats showed some interesting physical observation. Some of the animals gained weight (Obese group), while

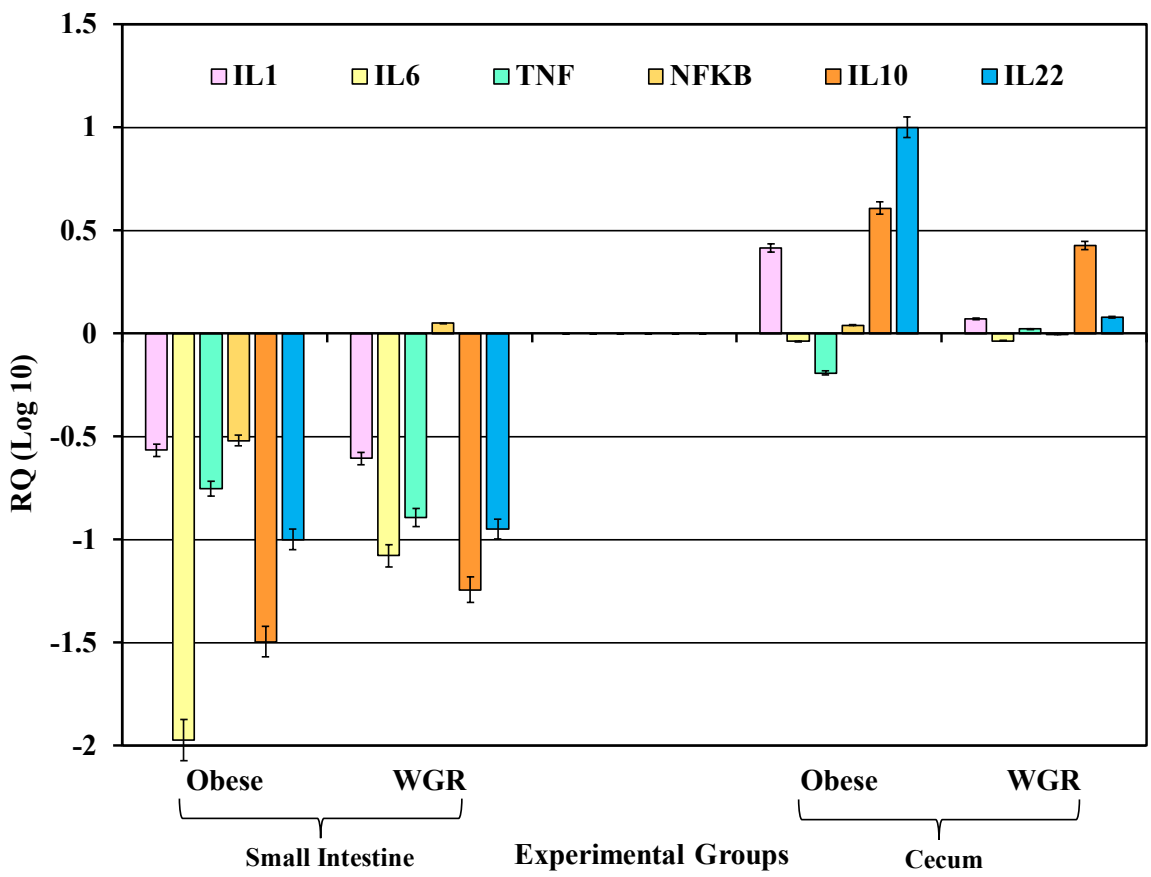

Figure 3. Gene expression analysis of inflammatory markers in cecum and small intestine samples of CAF diet and Normal chow diet fed SD rats. 
some of the animals are same as control animals without weight gain, and we designated them as Weight Gain Resistant (WGR groups). To explore the mechanism behind this physiological observation, this study proposed to analyze the serum biochemical parameters and inflammatory response due to diet induced gut microbial dysbiosis. After euthanasia, internal organs were observed during necropsy procedure. Accumulation of white and brown adipose tissue observed on different parts of the obese group. In addition, organ size in the obese group is comparatively larger than normal and WGR groups. The accumulation of fat in obese rat indicates that intake of high-energy diet and this may be associated with the physiological disorder [13].

Due to the continuous consumption of CAF diet, Glycemic index is comparatively higher in CAF diet fed groups and triglycerides level also elevated in the obese samples. With respect to the other lipid parameters, there is no much significant difference between the groups and it is significantly higher than the normal range. However, serum albumin is considered as a negative acute phase protein, and might be decreased during inflammatory conditions, such as obesity. In the present study, the amount of serum albumin decreased after CAF diet consumption in experimental animals. On the other hand, urea and liver enzymes activities (AST \& ALT) were analyzed to check their regulatory function and associated liver damage. The results revealed that consumption of CAF diet influenced the development of adiposity and alteration in metabolic parameters, which is associated with cardiovascular and physiological disorders [14].

The result of the serum parameters suggested that some of the experimental animals are metabolically healthy with obese body but few of them are metabolically unhealthy with normal body weight like normal and obese animals. More detailed and target oriented research efforts needed to address the mechanism behind these findings. More recent studies have revealed that gut microbiota are highly influenced by CAF diet and alters the metabolic signaling pathways which in turn impacts the energy balance and physiological mechanism [15] [16].

Although there were several studies reports about gut microbial dysbiosis and Firmicutes/Bacteroidetes at phylum level richness [17], however few of them discussed about abundance ratio at genera level. Hence, it is critical to explore the gut microbial dysbiosis at genera level to understand their impact on metabolism. Our previous research [6] reported that there are various level (Phylum, Order and Family) of microbial dysbiosis in the intestinal region. The present research highlights more on the shift in gut microbial proportion at genera level. The specific set of genera selected in this study were the ones showed interesting results at family level in our earlier report. Figure 2(a) and Figure 2(b) exhibits the relative abundance ratio in phylum and genera level. There is no great significant in the phylum level richness of Firmicutes/Bacteroidetes whereas in the genera level there is reasonable shift in CAF diet groups when compared with control groups. On the other hand, the percentage of Firmicutes increasing in the gut microbiota in obese animals (FP-O) and it is due to increased energy harvest from the diet and induce the absorption of more calories which leads to 
the obesity [18] [19].

To date many studies have reported the influence of high carb and fat diet and obesity on the level of specific phyla, with changes in the ratio of Firmicutes to Bacteroidetes which being reported in both human and rodents [20]. The same results we obtained in (CAF) diet experiment, while the increasing in the ratio of Firmicutes to Bacteroidetes level in cecum fecal samples was noticed. For the Clostridiales bacteria showed increasing in cecum fecal samples in CAF diet-fed animals more than WGR groups compared to the normal animal groups. On the other hand, increasing in Clostridiales and significantly increasing in Lactobacillales in obese groups in (CAD) diet fed rats [21]. While in other reports, they found that ratio of Firmicutes to Bacteroidetes was increased at species level [22]. However, other research reports showed that there is no change in Bacteriodetes at family or phylum level [23], but there is increase [24] and decrease in Firmicutes levels [25].

These results indicates that the CAF diet highly shapes the microbial diversity in gut region. Bacteroidetes-prevotella significantly increased in WGR samples. Several researches were reported that Firmicutes were greatly influenced by CAF diet [26] and in contrast, few of them reported Bacteroidetes [23] are increased significantly and some reports disclosed that there is no significant change in the Firmicutes/ Bacteroidetes ratio [18] [27]. Even though with all these considerable research findings, there is very limited research carried out to explore the plausible mechanism behind the gut microbial dysbiosis and still more research effort is needed to gain more knowledge.

Most research reports on diet induced gut microbiome change indicates that CAF diet alters the gut microbial population and induces the inflammatory response [28]. Increase in Gram-negative bacterial composition (Bacteroidetes) reflects in the LPS production, which subsequently cause inflammation in the gut region. Rainone et al. reported that the pathogenesis of obesity has a relationship with the presence of low-degree inflammation [4]. Moreover, variation in gut microbiota composition also affects the gut barrier function (Tight junction proteins) and increase metabolic endotoxemia, which in turn activate the innate immune system and low-grade inflammation [5] [29].

In this study, we investigated the effect of gut microbial dysbiosis on expression of inflammatory markers such as IL1 $\beta$, IL6, IL10, IL22, TNF and NfkB in the cecum and small intestine tissue samples of the CAF diet-fed rats. The results showed significant up-regulation of inflammatory cytokines such as, IL- $1 \beta$, IL-10 and IL-22 in both WGR and obese groups compared to the control groups whereas most of them are downregulated in small intestine samples irrespective of experimental diet regime. In summary, our research results demonstrate the interaction between CAF diet, gut microbiota and inflammation. The study show that CAF diet alters gut microbiota that might produce signaling components causing up-regulation of chemokines at the tissue level that might contribute in low-grade systemic inflammation related to CAF-diet induced obesity. 


\section{Conclusion}

Several studies have shown altered composition of the gut microbiota in the context of obesity in human and rodents and they conclude that all of these changes could contribute to the metabolic diseases. In our study, the analysis of gut microbiota community, distribution and its relation with metabolic measures in (SD) rats has highlighted that many bacterial species are altered in their ratio to other species in the gut microbiome and they are highly linked to development of obesity response to CAF diet. This observation indicates that the microbiota species could be a potential therapeutic target, such as Lactobacillus species, which can decrease in response of a CAF diet. Moreover, the modulation of gut microbiota is associated with development of inflammation, increase in the lipids and liver profiles parameter in CAF diet-fed rat. Further studies are warranted to explore and determine the mechanisms that regulate CAF diet induced obesity. In conclusion, understanding the mechanisms that linking the gut microbiota with obesity, and the knowledge on relevant compositional and functional characteristics of the gut microbiome, will pave the way for future research to develop more targeted approach aimed at restoring a more healthy gut microbiome to prevent obesity and related metabolic conditions.

\section{Acknowledgements}

The authors acknowledge Qatar University-VP for Research and Graduate studies for the financial support via faculty internal grant \#QU-G-CAS-BES-13/14-18) and the graduate assistantship provided to Mrs. Reem Moath Al Asmar.

\section{Conflicts of Interest}

The authors declare no conflict of interest.

\section{References}

[1] Rowland, I., Gibson, G., Heinken, A., Scott, K., Swann, J., Thiele, I. and Tuohy, K. (2018) Gut Microbiota Functions: Metabolism of Nutrients and Other Food Components. European Journal of Nutrition, 57, 1-24. https://doi.org/10.1007/s00394-017-1445-8

[2] Do, M., Lee, E., Oh, M.J., Kim, Y. and Park, H.Y. (2018) High-Glucose or -Fructose Diet Cause Changes of the Gut Microbiota and Metabolic Disorders in Mice without Body Weight Change. Nutrients, 10, 761. https://doi.org/10.3390/nu10060761

[3] Milagro, F.I., Campión, J. and Martínez, J.A. (2006) Weight Gain Induced by HighFat Feeding Involves Increased Liver Oxidative Stress. Obesity, 14, 1118-1123. https://doi.org/10.1038/oby.2006.128

[4] Rainone, V., Schneider, L., Saulle, I., Ricci, C., Biasin, M., Al-Daghri, N.M., Giani, E., Zuccotti, G.V., Clerici, M. and Trabattoni, D. (2016) Upregulation of Inflammasome Activity and Increased Gut Permeability Are Associated with Obesity in Children and Adolescents. International Journal of Obesity, 40, 1026. https://doi.org/10.1038/ijo.2016.26

[5] Gregor, M.F. and Hotamisligil, G.S. (2011) Inflammatory Mechanisms in Obesity. Annual Review of Immunology, 29, 415-445. 
https://doi.org/10.1146/annurev-immunol-031210-101322

[6] Varadharajan, K., Shanmugakonar, M., Das, S.C. and Al-Naemi, H.A. (2018) Gut Microbiota Dysbiosis in Cafeteria Diet Fed Sprague Dawley Rats. Advances in Microbiology, 8, 975-993. https://doi.org/10.4236/aim.2018.812066

[7] Marques, C., Meireles, M., Norberto, S., Leite, J., Freitas, J., Pestana, D., Faria, A. and Calhau, C. (2016) High-Fat Diet-Induced Obesity Rat Model: A Comparison between Wistar and Sprague-Dawley Rat. Adipocyte, 5, 11-21. https://doi.org/10.1080/21623945.2015.1061723

[8] Buyukdere, Y., Gulec, A. and Akyol, A. (2019) Cafeteria Diet Increased Adiposity in Comparison to High Fat Diet in Young Male Rats. PeerJ, 7, e6656.

https://doi.org/10.7717/peerj.6656

[9] Scoaris, C.R., Rizo, G.V., Roldi, L.P., de Moraes, S.M.F., de Proença, A.R.G., Peralta, R.M. and Natali, M.R.M. (2010) Effects of Cafeteria Diet on the Jejunum in Sedentary and Physically Trained Rats. Nutrition, 26, 312-320. https://doi.org/10.1016/j.nut.2009.04.012

[10] Sampey, B.P., Vanhoose, A.M., Winfield, H.M., Freemerman, A.J., Muehlbauer, M.J., Fueger, P.T., Newgard, C.B. and Makowski, L. (2011) Cafeteria Diet Is a Robust Model of Human Metabolic Syndrome with Liver and Adipose Inflammation: Comparison to High-Fat Diet. Obesity, 19, 1109-1117.

https://doi.org/10.1038/oby.2011.18

[11] de Castro Ghizoni, C.V., Gasparin, F.R.S., Júnior, A.S.M., Carreno, F.O., Constantin, R.P., Bracht, A., Iwamoto, E.L.I. and Constantin, J. (2013) Catabolism of Amino Acids in Livers from Cafeteria-Fed Rats. Molecular and Cellular Biochemistry, 373, 265-277. https://doi.org/10.1007/s11010-012-1499-0

[12] Gasparin, F.R.S., Carreño, F.O., Mewes, J.M., Gilglioni, E.H., Pagadigorria, C.L.S., Natali, M.R.M., Utsunomiya, K.S., Constantin, R.P., Ouchida, A.T., Curti, C. and Gaemers, I.C. (2018) Sex Differences in the Development of Hepatic Steatosis in Cafeteria Diet-Induced Obesity in Young Mice. Biochimica et Biophysica Acta (BBA) Molecular Basis of Disease, 1864, 2495-2509. https://doi.org/10.1016/j.bbadis.2018.04.004

[13] Heitmann, B.L. and Frederiksen, P. (2009) Thigh Circumference and Risk of Heart Disease and Premature Death: Prospective Cohort Study. British Medical Journal, 339, 3292. https://doi.org/10.1136/bmj.b3292

[14] Buyukdere, Y., Gulec, A. and Mutlu, A.A. (2018) Effect of Cafeteria Diet and High Fat Diet on Body Composition and Biochemical Parameters in Rats. Clinical Nutrition, 37, S269-S270. https://doi.org/10.1016/j.clnu.2018.06.1947

[15] MacQueen, H.A., Sadler, D.A., Moore, S.A., Daya, S., Brown, J.Y., Shuker, D.E., Seaman, M. and Wassif, W.S. (2007) Deleterious Effects of a Cafeteria Diet on the Livers of Nonobese Rats. Nutrition Research, 27, 38-47.

https://doi.org/10.1016/j.nutres.2006.10.003

[16] Mariné-Casadó, R., Domenech-Coca, C., Del Bas, J.M., Bladé, C., Arola, L. and Caimari, A. (2018) Intake of an Obesogenic Cafeteria Diet Affects Body Weight, Feeding Behavior and Glucose and Lipid Metabolism in a Photoperiod-Dependent Manner in F344 Rats. Frontiers in Physiology, 9, 639.

https://doi.org/10.3389/fphys.2018.01639

[17] Brooks, S.P., McAllister, M., Sandoz, M. and Kalmokoff, M.L. (2003) Culture-Independent Phylogenetic Analysis of the Faecal Flora of the Rat. Canadian Journal of Microbiology, 49, 589-601. https://doi.org/10.1139/w03-075

[18] Turnbaugh, P.J., Ley, R.E., Mahowald, M.A., Magrini, V., Mardis, E.R. and Gordon, 
J.I. (2006) An Obesity-Associated Gut Microbiome with Increased Capacity for Energy Harvest. Nature, 444, 1027. https://doi.org/10.1038/nature05414

[19] Krajmalnik-Brown, R., Ilhan, Z.E., Kang, D.W. and DiBaise, J.K. (2012) Effects of Gut Microbes on Nutrient Absorption and Energy Regulation. Nutrition in Clinical Practice, 27, 201-214. https://doi.org/10.1177/0884533611436116

[20] Murphy, E.A., Velazquez, K.T. and Herbert, K.M. (2015) Influence of High-Fat-Diet on Gut Microbiota: A Driving Force for Chronic Disease Risk. Current Opinion in Clinical Nutrition and Metabolic Care, 18, 515. https://doi.org/10.1097/MCO.0000000000000209

[21] Magnusson, K.R., Hauck, L., Jeffrey, B.M., Elias, V., Humphrey, A., Nath, R., Perrone, A. and Bermudez, L.E. (2015) Relationships between Diet-Related Changes in the Gut Microbiome and Cognitive Flexibility. Neuroscience, 300, 128-140. https://doi.org/10.1016/j.neuroscience.2015.05.016

[22] Collado, M.C., Isolauri, E., Laitinen, K. and Salminen, S. (2008) Distinct Composition of Gut Microbiota during Pregnancy in Overweight and Normal-Weight Women. The American Journal of Clinical Nutrition, 88, 894-899. https://doi.org/10.1093/ajcn/88.4.894

[23] Schwiertz, A., Taras, D., Schäfer, K., Beijer, S., Bos, N.A., Donus, C. and Hardt, P.D. (2010) Microbiota and SCFA in Lean and Overweight Healthy Subjects. Obesity, 18, 190-195. https://doi.org/10.1038/oby.2009.167

[24] Zuo, H.J., Xie, Z.M., Zhang, W.W., Li, Y.R., Wang, W., Ding, X.B. and Pei, X.F. (2011) Gut Bacteria Alteration in Obese People and Its Relationship with Gene Polymorphism. World Journal of Gastroenterology. WJG, 17, 1076-1081. https://doi.org/10.3748/wjg.v17.i8.1076

[25] Zhang, H., DiBaise, J.K., Zuccolo, A., Kudrna, D., Braidotti, M., Yu, Y., Parameswaran, P., Crowell, M.D., Wing, R., Rittmann, B.E. and Krajmalnik-Brown, R. (2009) Human Gut Microbiota in Obesity and after Gastric Bypass. Proceedings of the National Academy of Sciences, 106, 2365-2370. https://doi.org/10.1073/pnas.0812600106

[26] Koliada, A., Syzenko, G., Moseiko, V., Budovska, L., Puchkov, K., Perederiy, V., Gavalko, Y., Dorofeyev, A., Romanenko, M., Tkach, S. and Sineok, L. (2017) Association between Body Mass Index and Firmicutes/Bacteroidetes Ratio in an Adult Ukrainian Population. BMC Microbiology, 17, 120. https://doi.org/10.1186/s12866-017-1027-1

[27] Duncan, S.H., Lobley, G.E., Holtrop, G., Ince, J., Johnstone, A.M., Louis, P. and Flint, H.J. (2008) Human Colonic Microbiota Associated with Diet, Obesity and Weight Loss. International Journal of Obesity, 32, 1720-1724. https://doi.org/10.1038/ijo.2008.155

[28] Huttenhower, C., Gevers, D., Knight, R., Abubucker, S., Badger, J.H., Chinwalla, A.T., Creasy, H.H., Earl, A.M., FitzGerald, M.G., Fulton, R.S. and Giglio, M.G. (2012) Structure, Function and Diversity of the Healthy Human Microbiome. Nature, 486, 207-214. https://doi.org/10.1038/nature11234

[29] Levitan, E.B., Cook, N.R., Stampfer, M.J., Ridker, P.M., Rexrode, K.M., Buring, J.E., Manson, J.E. and Liu, S. (2008) Dietary Glycemic Index, Dietary Glycemic Load, Blood Lipids, and C-Reactive Protein. Metabolism, 57, 437-443. https://doi.org/10.1016/j.metabol.2007.11.002 